\title{
La expresión de la función semántica de Procedencia en las comedias de Plauto*
}

\section{Berta González Saavedra*}

\section{Resumen}

Desde que en los años ochenta la Lingüística Cognitiva puso el foco de atención en la semántica, el estudio de los casos ha recobrado un nuevo interés. En lo que al ablativo latino se refiere, su semántica ha sido estudiada desde antaño en listas inmensas de valores que resultan poco prácticas para entender su funcionamiento y no se pueden extraer los parámetros que determinan su significado. El presente artículo quiere evidenciar cuáles son estos parámetros cuando el ablativo latino expresa la Procedencia.

Palabras clave: Latín, sintaxis, Plauto, semántica, ablativo.

\section{The Expression of the Semantic Function 'Source' in Plautus Comedies}

\begin{abstract}
Since Cognitive Linguitics focused on semantics in the 8o's, the study of cases has regained interest for linguists. Regarding the Latin ablative, the tradicional studies have made up long lists of meanings with few clarity that make difficult to understand how this case works, and the parameters which determine its meaning cannot be comprenhensible. This study wants to evince which this parameters are, when the Latin ablative is used to express Source.
\end{abstract}

Keywords: Latin, syntax, Plautus, Semantics, ablative.

* Este trabajo forma parte del proyecto de investigación Estudicos de formación nominal: lenguas paleohispánicas e indoeuropeas antiguas (FFII 2009-13292-C03-02).

** Española. Doctora en Estudios del Mundo Antiguo de la Universidad Complutense de Madrid (España). bertagonzalezsaavedra@filol.ucm.es 


\section{Introducción}

Uno de los grandes aportes de la Lingüística Cognitiva fue la recuperación del interés por la semántica, disciplina lingüística que había quedado relegada a un segundo plano por la Lingüística Generativa (Luraghi, 2009). Para el estudio de las lenguas que presentan un sistema de casos, como el latín, el otorgar un significado a los morfemas permite enfrentarse a problemas que no se han resuelto, como su polisemia. El latín, así como el griego clásico, han sido objeto de innumerables estudios desde los principios de la propia reflexión lingüística, pero cuando la tradición se enfrentaba a la cuestión de la polisemia que presentan los casos, la solución tradicional ha sido la de elaborar largas listas en las que se recogían con profusión todos los significados atribuidos a cada uno de los casos. Con el surgimiento de la Lingüística Indoeuropea esta tendencia se agravó más, dado que en el funcionamiento de los casos se quiso ver la semántica original que se proponía para las marcas y de esta manera reconstruir los casos en la lengua indoeuropea (cf. entre otros Brugmann, 1892 y 1911).

En las últimas décadas la semántica de los casos latinos se ha estudiado desde perspectivas modernas, como la Gramática Funcional y Cognitiva, como se puede ver en la sintaxis coordinada por J.M. Baños (2009). Estos estudios incluyen de forma sistemática otros parámetros que antes no se habían concebido como determinantes, por ejemplo los marcos predicativos: J. de la Villa ya había apuntado la importancia de los marcos predicativos y la vinculación de los varios sintagmas que forman parte de la predicación como elementos requeridos obligatoriamente por el verbo o no (argumentos o satélites) a la hora de determinar su funcionamiento semántico. Además de eso, la naturaleza misma de las palabras declinables es otro de los factores a tener en cuenta: su posición en la Jerarquía de animación (Silverstein, 1986), y sus posibilidades de convertirse en tópico o tema del contexto lingüístico.

El objetivo de este artículo es presentar los datos que ofrece el ablativo singular en Plauto cuando expresa Procedencia, acompañado o no de preposiciones, con el fin de explicitar cómo los parámetros que he destacado en el párrafo anterior (marco predicativos y la naturaleza semántica de las palabras) determinan y justifican la presencia de las marcas, i.e.: ablativo con preposición o sin ella.

Para ello presentaré los datos que he recogido de las comedias de Plauto (excepto Vidularia, debido a su carácter fragmentario): ablati- 
vos singulares con y sin preposición de sustantivos comunes (extraídos del léxico de A. Maniet -1969-) con los verbos de los que dependen los sintagmas que expresan la Procedencia. Los predicados son analizados de acuerdo con la clasificación hecha en mi tesis doctoral (González Saavedra, 2015, p. 661-684) de los verbos latinos siguiendo el catálogo y las definiciones propuestas para el castellano por el proyecto ADESSE.

En este artículo manejo el concepto 'función semántica' de acuerdo con la definición que propuso E. Crespo (1997): nociones semánticas que todas las lenguas expresan, que son de carácter universal. En concreto, la función semántica de Procedencia con la que he trabajado en este artículo es la propuesta por él mismo "[E]l término designa normalmente una entidad espacial y expresa el origen de un movimiento o de una entidad (origen personal o material) o de una situación (o reacción)."

La estructura del artículo es la siguiente: 2. presentación de la expresión de Procedencia desde la perspectiva tradicional y desde la gramática Funcional-Cognitiva, 3. presentación y análisis de los datos recogidos en Plauto: ablativos sin preposición y ablativos con preposición, y 4. conclusiones.

\section{La expresión de la Procedencia en latín}

\subsection{El enfoque tradicional}

Para el enfoque tradicional esta es la función central que desempeña el ablativo separativo, de acuerdo con la división que se hace del caso: ablativo locativo, ablativo separativo y ablativo instrumental. En la mayoría de las gramáticas de enfoque tradicional se recogen listados de contextos en los que aparece esta función semántica, de acuerdo con las marcas utilizadas: solo el ablativo, el ablativo con $a b$, el ablativo con de y el ablativo con ex. En estas gramáticas, se hace una distinción de distintos tipos de ablativos separativos de acuerdo con la morfología verbal (si el verbo tiene o no un prefijo que indique separación) (Kühner-Stegmann, 1966, p. 362-364), con el sentido metafórico de dicha separación (Bennet, 1966, que distingue el ablativo separativo del de origen), y con el léxico (listas de sustantivos en ablativo y de verbos que rigen ablativo se encuentran en Bennet, 1966, por ejemplo). 


\subsection{El enfoque funcional-cognitivo}

Desde una perspectiva funcional-cognitiva para el latín se engloban en una misma función estos ablativos, denominados todos de Procedencia, y se atiende a los rasgos más prototípicos que podrían justificar su interpretación: verbos de movimiento o similares (movimiento abstracto), prefijados o no y un léxico que haga referencia a lugares (Torrego, 2009, p. 326 y 237). Además, hay que tener en cuenta que el ablativo entra en competencia con otras marcas (el ablativo acompañado de las preposiciones $a b, d e$ y ex) que sirven para denotar de manera más específica la Procedencia. Cuando las construcciones en las que aparece la Procedencia son menos prototípicas los límites con otras funciones se desdibujan y las interpretaciones de esta función pueden ser más abstractas (p. 326 y 327). En lo que se refiere al ablativo de Procedencia con sentido metafórico se incluyen los contextos en los que el desplazamiento, expresado por la acción verbal, no es real: 'nacer,' 'dar,' 'liberar,' 'defender,' 'comprar,' 'carecer,' etc. En estas ocasiones, se deben clasificar los verbos de acuerdo con su semántica, para ver si hay grupos de verbos que requieran con más frecuencia que otros la expresión de la Procedencia.

\section{Los datos que ofrece Plauto ${ }^{1}$}

\subsection{Los ablativos sin preposición}

En el corpus seleccionado he encontrado 177 ablativos sin preposición que expresan Procedencia. Del total, 65 son sustantivos que hacían referencia a lugares y 44 de ellos están regidos por verbos de Desplazamiento. Este es el contexto más esperable para encontrar ablativos de Procedencia; sin embargo, hay datos reveladores por ser menos esperables que cabe resaltar:

- En catorce ocasiones el verbo caveo (clasificado según el criterio de ADESSE con Actividad) aparece con sustantivos abstractos. Se trata de malum e infortunium, en una construcción que no es prototípica de la Procedencia, que se podría considerar cercana a una Causa: cave malo, 'cuídate del mal'.

1 Los datos están en las tablas L3, L4, L5 y L6 (González Saavedra, 2015, p. 92-116). 
- A pesar de que los verbos de Desplazamiento son los más frecuentes, también destacan los verbos de Vida (un total de veinticinco ablativos de Procedencia), entre los que se encuentran los que significan 'nacer' y sus sinónimos; en este grupo los sustantivos en ablativo no son topónimos, sino preferentemente sustantivos abstractos y humanos (dieciséis y seis pasajes respectivamente). También destacan los verbos de Posesión (catorce ablativos sin preposición) entre los cuales se hallan, predominantemente, los verbos que expresan la idea de 'carecer,' 'faltar', es decir, los antónimos de la posesión.

- En el corpus plautino hay trece pasajes en los que aparecen adjetivos con ablativos sin preposición que he clasificado con Procedencia. Para categorizar semánticamente estos adjetivos he seguido la propuesta de clasificación de Givón (2001, p. 81-83). Estos son fugitivus, que dentro de los adjetivos menos prototípicos, se incluye dentro de los Estados transitorios y, además, se trata de un adjetivo derivado a partir del verbo fugio, clasificado como Desplazamiento, con lo que la vinculación con complementos que indiquen Procedencia es análoga a la del verbo; prognatus, creado mediante un prefijo a partir del participio del verbo nascor (clasificado como Vida); cassus, dentro de los adjetivos menos prototípicos ${ }^{2}$, se incluye dentro de los Estados transitorios y está relacionado con la raíz del verbo $\mathrm{caedo}^{3}$, clasificado como Posesión; y expers, que, dentro de los adjetivos menos prototípicos, se incluye dentro de los Estados transitorios.

- Los cuarenta y cuarenta y dos pasajes restantes se corresponden a verbos de semántica más variada con movimiento implícito: verbos de Desplazamiento con partes del cuerpo (diez pasajes, véase el ejemplo Amph. 953) y con sustantivos abstractos (doce pasajes), y verbos de Adquisición (con abstractos en tres ocasiones).

Amph. 953

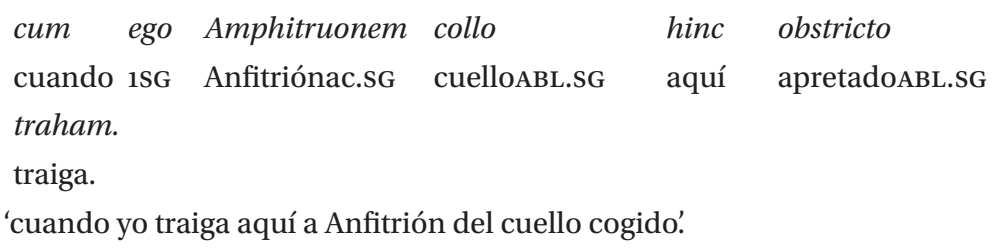

Siguiendo la nomenclatura utilizada por Givón (2001).

Según Ernout-Meillet (2001) s.v. 


\subsection{Los ablativos con preposición $a b$}

En las comedias de Plauto he encontrado 136 pasasajes en los que la preposición $a b$ acompaña un sustantivo y ambos expresan la Procedencia. En el comportamiento de estos sintagmas hay que llamar la atención sobre dos aspectos:

- Prevalecen los sustantivos concretos frente a los abstractos (solo en 23 ocasiones el sustantivo es abstracto), y dentro de ellos los que hacen referencia a humanos y a lugares ( 49 y 48 pasajes respectivamente). En el caso de los ablativos sin preposición, también prevalecen los concretos frente a los abstractos, aunque contados individualemente los concretos, prevalecen los abstractos, lo cual no sucede en los sintagmas introducidos por $a b$.

- Los verbos que con más frecuencia aparecen acompañados de $a b$ y ablativo con función Procedencia son los de Desplazamiento (73 ocasiones), seguidos de lejos por los de Adquisición (diecinueve ocasiones) y por sum (once pasajes).

- Llama la atención que esta preposición sea la elegida para expresar la Procedencia con sustantivos humanos, dado que la construcción de la preposición $a b$ con ablativo de persona es la que se atribuye tradicionalmente al 'agente'. Sin embargo, a partir de pasajes como As. 7o, se puede ver que hay una especialización en el uso de esta preposición para expresar la Procedencia con seres humanos.

As. 70

quam amabam abduxit ab lenone mulierem; la_queAc.sg quería ha_sacado PREP proxenetaABL.sg mujerAc.sG 'la mujer que yo quería la ha sacado de la casa del proxeneta'

- Los treinta y tres pasajes restantes se reparten entre verbos de semántica variada. De ellos destacan tres grupos de verbos donde el movimiento está implícito: Petición (cinco pasajes), Transferencia (cuatro pasajes) y Comunicación (cuatro pasajes, como en el ejemplo).

Amph. 967

$\begin{array}{lllll}\text { Tu gubernatorem } & a & \text { navi } & \text { huc evoca } & \text { verbis } \\ \text { 2SG timonelAC.SG } & \text { PREP naveABL.SG aquí llama palabrasABL.PL } \\ \text { meis } & \text { Blepharonem, } & & \\ \text { míasablpl } & \text { Blefarónac.sg } & & \end{array}$

'Tú llama ahora al timonel Blefarón desde la nave aquí con mis palabras' 


\subsection{Los ablativos con preposición de}

La presencia de esta preposición acompañando ablativos con función de Procedencia es mucho menor que la anterior: 69 ocasiones. Los datos reseñables son los siguientes:

- Llama la atención que sean los sustantivos abstractos los que prevalecen solo por detrás de los que hacen referencia a lugares: de 69 pasajes, veintisiete son sustantivos que hacen referencia a lugares y dieciocho son sustantivos abstractos.

- De nuevo los verbos de Desplazamiento son los que predominan a la hora de aparecer acompañados de estos sintagmas preposicionales (34 pasajes), seguidos, de lejos, por los de Adquisición (doce pasajes), y dentro de estos, parece que los que indican una dirección de arriba a abajo, marcados por el preverbio de-, llevan como marca preposicional también de (Trin. 482).

Trin. 482

$\begin{array}{lllll}\text { decedam } & \text { ego illi } & \text { de via, } & \text { de semita, } \\ \text { alejaré } & \text { 1SG aquelDAT.SG } & \text { PREP caminoABL.SG } & \text { PREP senderoABL.SG } \\ \text { de } & \text { honore } & \text { populi; } & & \\ \text { PREP } & \text { honorABL.SG } & \text { pueblogEN.SG } & & \end{array}$

'por él yo me alejaré del camino, del sendero, del honor del pueblo'

- De los veintitrés pasajes restantes, cinco corresponden a verbos de Transferencia (como en el ejemplo de Capt. 111), otros cinco al verbo sum y tres a verbos de Creencia (como en el ejemplo de Mil. 1423). Hay que notar que dentro de estos pasajes, sobre todo con sustantivos abstractos, son numerosos los usos en los que el sintagma tiene un sentido partitivo, como se puede ver en Capt. 111:

Capt. 111

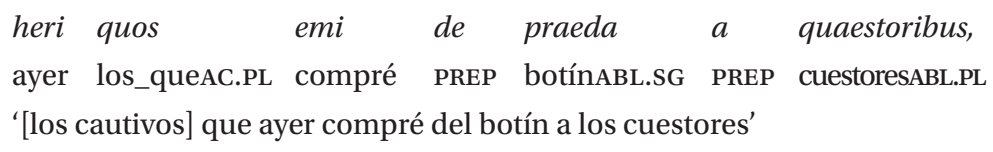

En este pasaje se pueden ver dos sintagmas preposicionales que indican Procedencia, pero con distinta marca, y de hecho aparecen yuxtapuestos. Por otra parte se puede ver que la preposición $a b$ es la que aparece preferentemente con seres humanos (los cuestores), mientras de en este caso está más cerca de la expresión de la partición. 
Mil. 1423

de tunica et chlamyde et machaera ne

prep túnicaabl.sg y mantoabl.sg y espadaabl.sg que_no

quid speres, non feres.

algoac.sg esperes no lleves

'que no esperes nada de la túnica, del manto y de la espada, que no los llevarás'

\subsection{Los ablativos con preposición ex}

Esta preposición es muy frecuente en la expresión de la Procedencia en Plauto, casi tanto como el ablativo sin preposición: 167 pasajes en total. De estos, además de la cantidad son reseñables los siguientes aspectos:

- El número de sustantivos abstractos es superior al de sustantivos que hacen referencia a lugares (61 pasajes y 54 respectivamente), como ocurre con el ablativo sin preposición.

- En cuanto a los verbos, siguen siendo más frecuentes los de Desplazamiento (81 pasajes), seguidos de facio (doce pasajes, como se ve en el ejemplo de Truc. 134) y sum (diez pasajes).

Truc. 134

nunc mulier facta est iam ex viro:

ahora mujerNom.sg hecha ha_sido ya PREP hombreABL.SG

'ahora la mujer ya ha sido hecha a partir del hombre'

- De los 64 pasajes restantes destacan los verbos en los que hay un movimiento implícito: Transferencia (en siete pasajes), Vida (seis pasajes), Adquisición (cinco pasajes), Petición (cinco pasajes), Modificación (cuatro pasajes) y Percepción (cuatro pasajes, como en el ejemplo de Rud. 1019).

Rud. 1019

$\begin{array}{lcccl}\text { At } & \text { ego } & \text { inspectavi } & e & \text { litore. } \\ \text { pero } & \text { 1SG } & \text { he_examinado } & \text { PREP } & \text { litoralABL.SG } \\ \text { 'pero yo he examinado desde el litoral' } & \end{array}$

\subsection{Los ablativos con preposición sub}

Un único pasaje aparece en el corpus plautino con función de Procedencia introducido por la preposición $s u b$. El verbo que rige el sintagma es de Desplazamiento, y esto es razón suficiente para la interpretación de este 
ablativo. Este uso de sub para expresar Procedencia es común y sería un significado antónimo al de la preposición de, que expresaría la dirección contraria del movimiento. El pasaje en cuestión es el siguiente:

Capt. 730

$\begin{array}{lllll}\text { interdius } & \text { sub } & \text { terra } & \text { lapides } & \text { eximet: } \\ \text { de_día } & \text { PREP } & \text { tierraABL.SG } & \text { piedrasAC.PL } & \text { saca }\end{array}$

'durante el día saca piedras de debajo de la tierra'

\section{Discusión de los datos y conclusiones}

A la luz de los datos que ofrece el corpus plautino y que se han expuesto en este artículo, se puede ver que no existen normas rígidas para la expresión de la Procedencia en el registro de latín que reflejan estos textos, al menos de forma similar a las que se han propuesto tradicionalmente para la lengua latina de época clásica.

En lo que se refiere a la expresión de la Procedencia, se puede ver lo siguiente:

1. Esta función aparece con mayor frecuencia con verbos que implican Desplazamiento, como era de esperar, ya que son los contextos en los que se expresa movimiento en los que la Procedencia y la Dirección aparecen en más ocasiones. Otros verbos en los que el movimiento es metafórico, como los verbos de Vida, Posesión, Adquisición (y sus antónimos), Transferencia e incluso Modificación son los que pueden aparecer acompañados de esta función bastantes veces.

2. Sin embargo, no se puede hablar de una restricción contextual marcada por los verbos. De hecho, se podría pensar que los verbos prefijados o los que indican movimiento serían los que regirían el ablativo sin preposición para expresar dicha función, pero los datos revelan que esto no es del todo cierto, pues ya se ha visto que la preposición de aparece en ocasiones remarcando el valor que aparece en el prefijo de- del verbo (Capt. 111) o la preposición sub refuerza el verbo eximo (Capt.730). El énfasis en esta función, porque se quiere añadir una determinación en la dirección del movimiento, es lo que lleva a la reiteración de la marca mediante preverbio, marca casual y preposición.

3. Hay que destacar, por otra parte, que los verbos clasificados como Posesión aparecen únicamente acompañados por ablativos sin preposición, y esto puede deberse a una lexicalización del ablativo con 
estos verbos anterior a la necesidad del refuerzo de la marca para la expresión de la Procedencia, aunque sería necesario un estudio en profundidad de estos verbos.

4. En cuanto a la clasificación léxica de los sustantivos que expresan esta función, se puede afirmar, que, como era de esperar, son los sustantivos que expresan lugar los que con mayor frecuencia desempeñan la Procedencia.

5. Es reseñable, sin embargo, que sustantivos cuyo referente es humano (como leno, 'proxeneta') desempeñen preferentemente también esta función con la marca tradicionalmente atribuida a la función de 'agente' $(a b)$, sea cual sea la clasificación verbal (As. 70), mientras que la preposición ex no aparezca, más que en contadas ocasiones y nunca con verbos clasificados como Desplazamiento (ningún sustantivo humano aparece introducido por ex con un verbo de Desplazamiento).

6. Otros aspectos remarcables son que los sustantivos que expresan lugar y los abstractos aparecen en más ocasiones en ablativo sin preposición que con preposición, esto puede deberse a que aparecen en contextos en los que la acción verbal de la que dependen es suficientemente clara para que la interpretación de los ablativos como Procedencia no necesite de ninguna marcación más.

7. En cuanto a las preposiciones y su distribución se podría pensar que la preposición $a b$ se está especializando en los sustantivos animados, en concreto con los humanos, (y en otras funciones, como la de 'agente') mientras la preposición ex es la menos marcada (frente a lo propuesto por Rubio, 1966: 177, donde afirma que de es la menos marcada de las tres). Además, la preposición de parece tener en algunas ocasiones un sentido partitivo, que también tiene ex, aunque con menos frecuencia, como se puede presumir del hecho de que de con sustancias aparece en más ocasiones que ex (siete ocasiones frente a dos). Sin embargo, de parece estar especializada para la expresión de la Referencia en los textos de Plauto (González Saavedra, 2015: 381-396).

En cuanto a las futuras investigaciones que se pueden hacer en este ámbito y que arrojarán sin duda nueva luz hay que incluir sin duda el análisis de los datos que aporten los ablativos plurales y los de los pronombres personales, para ver su distribución con preposición y sin ella, y ver si siguen los mismos parámetros que apuntan los ablativos singulares incluidos en este estudio. Su inclusión no es sencilla, dada la coincidencia 
en la marca con el dativo plural en el caso de los ablativos plurales y con el acusativo singular, en el caso de los pronombres personales en singular. Estos datos podrían confirmar lo que aquí se ha reseñado o indicar otros factores que no se han tenido en cuenta en este estudio, porque los datos que he elegido no los mostraban.

Asimismo, los pronombres personales pueden determinar la evolución hacia la marcación de las funciones semánticas de acuerdo con la Jerarquía de animación y las marcas de función que propuso F. Villar (1989). Estos datos, sumados a los que proporcionan las lenguas romances podrían dar un panorama extenso de la evolución de las marcas preposicionales usadas para la expresión de la Procedencia, que ayudarían a comprender el hecho de que preposiciones compuestas a partir de otras preposiciones (como desde en español o da en italiano) sean las que han prevalecido en las lenguas modernas para la Procedencia.

\section{Bibliografía}

ADESSE Base de datos de Verbos, Alternancias de Diátesis y Esquemas Sintáctico-Semánticos del Español, Universidad de Vigo. http://adesse.uvigo.es/

Baños Baños, J. M. (coord.) (2009), Sintaxis del latín clásico, Madrid: Liceus E-excellence.

Bennett, E. C. (1966), Syntax of Early Latin, II, Hildesheim, Zúrich-Nueva York: Georg Olms Verlagsbuchhandlung.

Brugmann, K. (1892), Compartive Grammar of the Indo-Germanic Languages, vol. III parte II, Nueva York: B. Westermann and Co. . (19112), Vergleichende Laut-, Stammbildungs- und Flexionslehere nebst lehre vom Gebrauch der Wortformen der indogermanischen Sprachen, vol. II parte III Estrasburgo: Karl J. Trübner.

Crespo Güemes, E. (1997), Sintaxis de los elementos de relación en griego clásico, en Actas del IX Congreso Español de Estudios Clásicos, Madrid: Ediciones Clásicas, 1-40.

Ernout, A. y Meillet A. (2001), Dictionnaire étymologique de la langue latine, París: Klincksieck.

Givón, T. (2001), Sytax: an Introduction, vol.1 Ámsterdam-Filadelfia, John Benjamins Publishing Company. 
González Saavedra, B. (2015), La expresión de la Procedencia en tres lenguas indoeuropeas antiguas: latín, griego e hitita, tesis doctoral disponible en http://eprints.ucm.es/32775/1/T36269.pdf

Kühner, R. y Stegmann, C. (1966=1912), Ausführliche Grammatik der lateinischen Sprache, zweiter Teil: Satzlehre, Hannover: Verlag Hahnsche.

Luraghi, S. (2009), "Case in Cognitive Grammar", en A. Malchukov y A. Spencer (eds.), The Oxford Handbook of Case, Oxford: Oxford University Press, 136-150.

Maniet, A. (1969), Plaute: lexique inverse, listes grammaticales, relevés divers, Hildelsheim: Olms.

Silverstein, M. (1986 reed.), Hierarchy of Features and Ergativity, en Muysken, P. y Riemsdijk, H. van, Features and Projections, De Gruyter: Dortrecht - Riverton, 163-232.

Rubio Fernández, L. (1966), Introducción a la sintaxis estructural del latín, vol. 1 Casos y preposiciones, Barcelona: Ariel.

Torrego, E. (2009), VIII. Ablativo, en J. M. Baños (coord.), 211-250.

Villa, J. de la (2003), "Límites y alternancias en los marcos predicativos", Praedicativa. Complementación en griego y latín, en J. M. Baños et al. (eds.), Santiago de Compostela: Universidad, 19-49.

Villar, F. (1989), Jerarquía y marcas de función, Salamanca: Ediciones Universidad de Salamanca. 